\title{
Migrazione alla ricerca di un'identità in La stagione della migrazione al nord di al-Ṭayyib Șāliḥ
}

Paolo La Spisa

\section{Introduzione}

Nell'immaginario europeo di matrice romantica, l'Oriente ha sempre rappresentato una meta privilegiata per scrittori e poeti. Goethe, Nerval, Burton sono solo alcuni dei nomi più celebri che in un modo o in un altro hanno subito la fascinazione dell'Oriente. Se da una parte questa fu alimentata da una certa letteratura orientalistica che nel XIX secolo occupava in modo sempre più massiccio gli scaffali delle più illustri istituzioni accademiche europee, ciò che più attirava delle terre d'Oriente erano le esperienze di viaggio, il cui scopo privilegiato era la ricerca di una nuova dimensione spirituale ed esistenziale da parte di artisti e scrittori. Chateaubriand e Lamartine prima di intraprendere i loro viaggi si erano già potuti creare una loro idea di Oriente che non di rado si scontrerà con una ben diversa e spesso, ai loro occhi, barbara realtà. Come ebbe a scrivere Edward Said nel suo celebre Orientalism: «In realtà, ciò che gli importa dell'Oriente è ciò che esso produce in Chateaubriand, le possibilità che dischiude al suo spirito, quel che gli permette di capire di sé stesso, delle sue idee e aspettative» (Said 1999, 174). In Nerval e Flaubert si concretizza quel gusto estetico tutto romantico in cui si fondono il fascino del macabro, tendenze e amori sadomasochistici e donne fatali (Praz 1966). L'Oriente dunque, dopo essere stato oggetto di conquista militare durante l'impresa coloniale, diviene luogo di conquista erotica, dove l'una si fa metafora dell'altra. Tuttavia, Nerval

\footnotetext{
Paolo La Spisa, University of Florence, Italy, paolo.laspisa@unifi.it, 0000-0001-9989-9279 FUP Best Practice in Scholarly Publishing (DOI 10.36253/fup_best_practice)

Paolo La Spisa, Migrazione alla ricerca di un'identità in La stagione della migrazione al nord di Al-Tayyib Sālih, pp. 319-341, (C) 2021 Author(s), CC BY 4.0 International, DOI 10.36253/978-88-5518-467-0.25, in Michela Graziani, Lapo Casetti, Salomé Vuelta García (edited by), Nel segno di Magellano tra terra e cielo. II viaggio nelle arti umanistiche e scientifiche di lingua portoghese e di altre culture europee in un'ottica interculturale, (c) 2021 Author(s), content CC BY 4.0 International, metadata CC0 1.0 Universal, published by Firenze University Press (www.fupress.com), ISSN 2704-5919 (online), ISBN 978-88-5518467-0 (PDF), DOI 10.36253/978-88-5518-467-0
} 
nel suo Voyage en Orient dichiarerà la sua intenzione di unirsi ad una donna araba, figlia di una terra considerata madre dell'umanità, non tanto per spirito di conquista, bensì per identificarsi con un altro-da-sé, alla ricerca di un'esperienza estraniante (Said 1999, 184).

Da un'altra prospettiva, l'Europa in quanto continente simbolo di quella categoria tutta culturale nota come Occidente, ha rivestito un ruolo di primaria importanza nell'immaginario arabo. A questo proposito è interessante porsi la domanda sulla funzione che hanno assunto il viaggio e la migrazione nella costruzione di questo immaginario. Potremmo in prima istanza affermare che nella storia dell'Oriente arabo e dell'Islam in generale, la migrazione e il viaggio rivestono un ruolo decisivo che va a costituire uno spartiacque tra ignoranza ed era islamica, tra fede e miscredenza. In epoca preislamica, tra V e VI secolo della nostra era, quando i poeti cominciarono a decantare gli aridi spazi d'Arabia e le gesta della propria tribù o di un parente scomparso in battaglia, la dimensione del viaggio acquista un ruolo centrale. Sebbene negli antichi componimenti poetici non si faccia riferimento alcuno all'itinerario né tanto meno alla sua destinazione, il viaggio nel deserto con i pericoli che esso comporta, diviene presto un vero topos poetico-letterario all' interno del quale vengono messi in risalto il coraggio del poeta e le sue virtù. Viaggio dunque, come prova esistenziale e lotta per l'esistenza (Jacobi 1982, 5), che diviene migrazione allorquando il poeta, rifiutato dai suoi contribuli, è costretto a vagare errabondo nel deserto in cerca di protezione dopo aver conosciuto l'esperienza del ripudio. L'altro e l'altrove sono i pilastri della dimensione esistenziale anche del musulmano, suggellata dal rito del pellegrinaggio a Mecca, luogo in cui si fondono le dimensioni spazio-temporali dell'hic et nunc per diventare eterne e assolute. Le origini dell' Islam affondano le proprie radici nell'esperienza del ripudio, del misconoscimento e quindi della migrazione. Dall'Ismaele biblico, a cuil'intera Umma islamica si richiama, al profeta arabo Muhammad, la migrazione (hiğra) è un'esperienza centrale che rappresenta il vero perno da cui far cominciare una nuova era (Benslama 2002, 124-9).

A questa eredità per dir così ancestrale, si aggiunge l'esperienza di rinnovamento e rinascita che tutto il mondo arabo visse tra Otto e Novecento e che va sotto il nome di Nahda (rinascita), grazie alla quale la dimensione del viaggio assunse un ruolo centrale anche dal punto di vista delle nuove sperimentazioni letterarie che in quegli anni si andavano profilando. Sull'onda del riformismo e della modernizzazione di molti paesi (Egitto, Siria e Libano in primis) furono previsti viaggi studio e di specializzazione in Europa al fine di formare le future classi dirigenti e impiegatizie di paesi ancora fin troppo appesantiti dall'eredità ottomana. Dal punto di vista delle ricadute artistico-letterarie, l'esperienza del viaggio di formazione, realmente vissuta dai primi scrittori e intellettuali arabi di epoca moderna, foggerà il genere romanzo, tradizionalmente inaugurato dalla pubblicazione di Zaynab (1913) di Muḥammad Husayn Haykal. Ecco allora che il viaggio, l'esperienza dello straniamento, dell'alienazione e in ultima analisi della scissione dell'Io del protagonista, acquisteranno una posizione centrale all'interno del nuovo genere letterario che ebbe il suo abbrivio con la prima generazione di romanzieri arabi (Casini et al. 2013). 
Queste premesse non sembrano anodine anche per chi si accinga a leggere un classico che ha rappresentato un vero punto di svolta nella letteratura araba contemporanea. Mawsim al-hiğra ilā šimāl [La stagione della migrazione a Nord, 1967] dello scrittore sudanese al-Ṭayyib Muhammad Șāliḥ Aḥmad (1929-2009) (Amyuni 1998), è un romanzo che non si presta facilmente a incasellamenti ermeneutici precostituiti. Studi post-coloniali, di genere e letture psicoanalitiche si sono moltiplicate nella letteratura critica del secolo scorso, proponendo spesso visioni 'a senso unico', quando è ben noto che ciò che fa assurgere un'opera letteraria al rango dei classici è la sua 'polisemia' e molteplicità di piani di lettura e di interpretazione. Una certa critica ha voluto porre il più noto romanzo di Șāliḥ in dinamica dialogica con un classico della letteratura europea di fine Ottocento. Cuore di tenebra di Joseph Conrad avrebbe rappresentato il termine di paragone a cui lo scrittore sudanese avrebbe opposto una forte risposta, laddove il colonizzato 'conquista' il colonizzatore e consuma così la sua vendetta (Shaheen 1985; Said 2001, 110-1; Mayer 2001, 141-2). La lettura post-coloniale del romanzo, sposata dalla maggior parte della critica sia europea che araba (Bakkār 2015), se pur lecita rischia di non cogliere il messaggio profondo che l'opera veicola. Come è stato giustamente sottolineato, l'invasione inglese in Sudan e lo scontro tra Sud e Nord rappresentano il sottofondo su cui la vera storia si dipana (Siddiq 1978, 67). Se da una parte i romanzi delle prime generazioni di scrittori come Țāhā Ḥusayn, Tawfīq al-Ḥakīm e Yahyaa Ḥaqqī, avevano posto al centro la questione del nazionalismo indipendentista o del viaggio di formazione in cui trovava ampio spazio il tema della contrapposizione tra Oriente e Occidente, modernità e tradizione, secolarismo e Islam, in al-Ṭayyib Șāliḥ le stesse tematiche vengono reinvestite di nuove funzioni retoriche, di nuove strategie narrative che si fanno veicoli di dinamiche intime ed esistenziali, in cui si dà voce alla crisi del soggetto in quanto soggetto. Perché, se è vero che l'autore si nasconde in modo ineffabile dietro i suoi personaggi e in ultima analisi dietro la sua arte, è altrettanto vero che i personaggi richiamano al lettore il problema del soggetto in un gioco continuo di rispecchiamenti e identificazioni. Si aggiunga inoltre che alla complessa questione dei rimandi inter-, iper- e para-testuali insiti in una qualsiasi opera letteraria (Genette 1997), si affianca quella dei limiti interpretativi da parte del critico e quindi la necessità di distinguere tra intentio operis e intentio lectoris (Eco 2016). Date simili premesse, in questo contributo sarà elaborata una proposta interpretativa che tiene conto delle quattro dimensioni in cui a ben vedere ogni testo letterario prende vita. Verrà tenuto conto del testo e della sua struttura cronotopica, dei personaggi e delle loro dinamiche psicologico-esistenziali, del lettore col suo immaginario e la sua 'enciclopedia' e infine della relazione tra le prime tre. Testo, personaggi, lettore e relazione sono le quattro dimensioni che costituiranno l'impianto euristico attraverso cui proporremo una lettura di questo classico della letteratura araba. Non diversamente si è recentemente espresso Antonio Pioletti interrogandosi sul rapporto tra opera artistica e realtà, e sulla funzione che lo spazio letterario all'interno della categoria bachtiniana del cronotopo assume in questa complessa relazione. 
La raffigurazione [dello spazio], inscindibile dalla dimensione temporale, non è certo il rispecchiamento del reale, ma il disvelamento della visione dialogica nei confronti del reale di cui il testo, che include autore, personaggi e destinatari, si fa vettore (Pioletti 2014, 28).

Poiché ogni impresa ermeneutico-interpretativa non può non confrontarsi con la questione del rapporto tra finzione artistica e realtà, non sarà inutile rievocare ciò che a tale proposito scriveva Bachtin. Distinguendo tra mondo raffigurato e mondo raffigurante, Bachtin metteva in guardia da facili semplificazioni che pongono tra le due raffigurazioni confini invalicabili.

Per quanto distinti tra loro siano il mondo raffigurato e quello raffigurante, per quanto immancabile sia la presenza di un confine rigoroso tra di essi, essi sono indissolubilmente legati tra loro e si trovano in un rapporto di costante azione reciproca, simile all'ininterrotto metabolismo tra l'organismo vivente e l'ambiente che lo circonda: finché l'organismo è vivo, esso non si fonde con questo ambiente, ma se lo si stacca dall'ambiente, esso muore (Bachtin 1979, 401).

L'immagine tolta dalla biologia chiarisce il rapporto 'fisiologico' che si instaura tra creazione artistica e realtà di cui il testo si fa interprete. In una simile prospettiva, il personaggio letterario acquisterà di senso per il lettore solo se diventa 'tridimensionale', ovvero realistico e verosimigliante, permettendo così l'instaurarsi di una tensione dialogica tra lettore e personaggio fatta di rispecchiamenti e identificazioni che vivificano l'opera stessa. A questo proposito, non sarà inutile prendere ispirazione dalla prospettiva relazionale adottata anche in altre discipline, come la fisica (Rovelli 2020) o la psicoanalisi di scuola kleiniana-bioniana (Ogden 1999).

\section{Il testo e la trama}

Come è stato detto, il viaggio di Nerval in Oriente era finalizzato alla ricerca di un'esperienza estraniante tramite l'unione erotica con una donna egiziana. Questo aspetto è particolarmente interessante per chi si accosti alla lettura di molti romanzi arabi di formazione, come Adīb di Ṭāhā Husayn ${ }^{1}$ o Qindīl Umm Hāšim [La lampada di Umm Hāšim] di Yaḥyā Haqqī. La stagione della migrazione al Nord si situa dunque all'interno di una tradizione letteraria ben precisa che ebbe inizio in Egitto nei primi decenni del Novecento. Il romanzo più noto dello scrittore sudanese, il cui profilo biografico riflette l'esperienza della formazione in Inghilterra dei suoi personaggi, è principalmente la storia della ricerca della vera identità del protagonista Mușțafā Sa ìd, personaggio a cavallo tra due mondi con cui egli intrattiene rapporti non privi di ambiguità e ambivalenza. Il racconto ha una struttura autodiegetica (Genette 1976, 291-6), dove il narratore interno partecipa alla storia che racconta; egli riveste il ruolo di detective sul conto di Mușțafā Sa 'īd con il quale instaura un meccanismo di identificazione-sdoppiamento. Il romanzo presenta contrazio-

Di questo romanzo si segnala la recente traduzione italiana di Maria Elena Paniconi, cfr. Husayn 2017. 
ni e dilatazioni spazio-temporali che strutturano un intreccio di stampo post-modernista. Data la complessità della trama, ne delineiamo qui brevemente la storia.

Il narratore, di cui non verrà mai rivelato il nome, torna in Sudan, nel suo villaggio di origine, dopo sette anni di permanenza in Inghilterra per motivi di studio. Tra gli abitanti che lo accolgono si accorge della presenza di un volto non noto. Chiede notizie ai parenti: è un forestiero venuto da Khartum cinque anni prima, il suo nome è Muștafā Sa î̀d. I due si incontrano e parlano in privato, il narratore chiede a Muștafā di rivelargli la sua vera identità. Segue il racconto in prima persona della storia di Muștafā Sa ìd. Egli rivela di aver avuto una brillante carriera che lo ha portato a Londra, dove ha conosciuto molte donne del luogo con cui ha vissuto avventure e relazioni sentimentali. Si sposa con Jean Morris. Viene condannato dalla Corte di Assise di Londra a sette anni di carcere per l'omicidio della moglie. Dopo aver scontato la pena, torna in Sudan per ricominciare una nuova vita, si sposa con una donna sudanese e ha due figli. Mușțafā Sa 'îd muore disperso durante un'inondazione del Nilo. Prima della sua scomparsa, Muștafā Sa ìd scrive una lettera indirizzata al narratore: gli affida la casa e lo nomina tutore dei figli. Lo invita a entrare nella stanza chiusa a chiave di casa sua, in cui troverà la risposta alle sue domande. Il narratore è vittima di pensieri ossessivi e ricorsivi che lo riconducono alla storia di Mușțafā. Wadd al-Rayyes, anziano proprietario terriero, vuole sposare la vedova di Muṣtafā Sa ìd e chiede al narratore di farsi da tramite. Tutto il capitolo è incentrato sulle capacità sessuali di Wadd al-Rayyes nonostante la sua avanzata età. Si fa evidente il contrasto tra la tracotanza sessuale del personaggio e la riservatezza e introversione del narratore. Il narratore rende visita alla vedova di Muștafāa Sa î̀d, sperando di carpire qualche informazione in più sul passato del marito scomparso. La vera identità del marito è ignota anche alla moglie Ḥusnā, la quale confessa di non essere mai entrata nella stanza dai «mattoni rossi». Il narratore, parlando con un vecchio amico di scuola, confessa di essere innamorato di Ḥusnā Bint Maḥmūd, vedova di Muștafāā Sa i ìd. La voce narrante torna a Khartum attraversando il deserto. Durante una tappa notturna, viene improvvisata una festa con $i$ beduini del luogo. La voce narrante ricorda a sé stesso che sua figlia si chiama Amal, speranza. L'io narrante torna al villaggio dopo sette mesi di lavoro a Khartum; apprende della morte di Wadd alRayyes e della moglie Husnā, vedova di Muștafā Sa ìd, la quale, dopo aver evirato e ucciso il secondo marito, si suicida. Il narratore entra finalmente nella stanza segreta di Muștafā Sa î̉j; uscito di casa, si getta nelle acque del Nilo.

Poiché sono molte le letture a cui una simile trama si presta, è utile chiarire che qui si è scelto di analizzare i due personaggi principali, il narratore e Muṣțafā Sa ìd, le dinamiche che essi instaurano tra di loro e con il mondo femminile.

\section{II narratore e l'esperienza del ritorno}

«Tornai alla mia gente, signori miei, dopo una lunga assenza»². Questo l'incipit del romanzo: una voce narrante in prima persona, che nel corso della storia

2 I riferimenti al testo si basano sulla seconda edizione della traduzione italiana a cura di Francesco Leggio. Il testo arabo di cui mi sono servito è quello dell'edizione stampata a 
si rivolge di tanto in tanto ai lettori con l'appellativo «signori miei». Da subito il lettore capisce di essere alle prese con un Bildungsroman arabo dalla struttura rovesciata, dove il racconto prende avvio con un ritorno anziché una partenza. La storia di un ritorno dopo l'esperienza dell'assenza ( $\dot{g} a y b a)$ indica un particolare rapporto col tempo e con lo spazio narrativo: il punto di vista spazio-temporale è quello del qui e ora; il narratore, tornando, sente di essere stato assente dal suo villaggio di origine, dalla sua famiglia, dalla sua gente. Ciò è confermato dalla frase successiva, in cui è utilizzato il verbo della stessa radice della parola «assenza»: wa-ḡâba 'annì l-kațìr, «e molto mi sono lasciato sfuggire». L'ottica dunque dell' io narrante non è quella di chi vuole raccontare al lettore la sua vita trascorsa in Inghilterra, bensì ciò che gli accade a partire dal momento del suo ritorno. L'attimo viene posto in relazione con i sentimenti e le emozioni ad esso correlati: «quando arrivai fu un momento meraviglioso».

Sin dal primo capitolo, inoltre, si delinea la dinamica speculare tra l'io narrante e Mușțafā Sa î̀, il vero protagonista della storia. La domanda su chi realmente sia Mușțafā si pone invero sin dall'inizio del romanzo e crea una tensione narrativa e un effetto suspence che solo alla fine troverà una sua risoluzione. I due momenti decisivi del romanzo sono dunque il II capitolo, in cui il protagonista racconta la sua storia al narratore parlando in prima persona (la stessa della voce narrante) e il capitolo VIII, in cui il narratore, dopo la morte di Mușțafā Sa ìd e di sua moglie, entra nella stanza segreta e viene così rivelata al lettore la vera storia del protagonista. Tra questi due momenti si crea un climax, il tempo si dilata ma non per questo diminuisce di senso né si svuota. La voce narrante è ossessionata dalla presenza di Muștafā Sa ĩd che si fa via via sempre più incalzante ad ogni incontro, in ogni luogo, sino a culminare nel punto apicale del romanzo: la stanza segreta.

L'elemento che fa sì che il narratore assuma un atteggiamento indagatorio nei riguardi di Mușțafā Sa ìd è la sua recitazione di alcuni versi di poesia in lingua inglese. Dietro quell'uomo dai modi raffinati si deve nascondere una storia.

Le orecchie mi avevano forse ingannato la notte scorsa? La poesia inglese che aveva recitata era una realtà. [...] Forse quello ha ucciso qualcuno da qualche parte ed è evaso di prigione... Forse... Ma che segreti possono esserci in questo paese? (Salih 2011, 38).

La poesia in inglese rimanda a un passato nascosto, sorge immediato il dubbio sulla moralità del protagonista; è come se il narratore si domandasse: $<\grave{E}$ forse un assassino venuto a nascondersi tra la mia gente? Dopotutto, che segreti possono mai essere nascosti in questo paese, nel mio paese?», sono le questioni che d'ora in avanti assilleranno l'io narrante. L'ultima domanda è di basilare importanza perché implicano il rapporto viscerale che il narratore ha col proprio paese di origine che egli dichiara più volte di conoscere alla perfezione.

Tunisi nel 2015 dal Dār al-ğanūb li-l-našr, con una presentazione di Tawfīq Bakkār e illustrazioni di Ḥasanayn Ibn 'Amū. 
D’altronde, l'abbiamo visto, il suo scopo è narrare il ritorno e non la partenza. Le prime pagine del romanzo si profondono in descrizioni naturalistiche del villaggio. Un panorama agreste, di una campagna che non aveva mai dimenticato e che è rimasta immutata.

Udii il tubare delle tortore e guardando attraverso la finestra la palma ritta nell'atrio di casa nostra, compresi che la vita andava ancora bene. Ne rimiravo il tronco forte e diritto, le radici piantate in terra, le palme verdi pendenti su in cima; e provavo un senso di serenità. Sentivo che non ero una piuma in balìa del vento, ma ero come quella palma: una creatura con un'origine, con delle radici, con uno scopo (Salih 2011, 26).

Il narratore è appena tornato nel suo villaggio natale in riva al Nilo e comincia a riprendere contatto con il suo mondo, le sue origini, la sua famiglia e gli abitanti del villaggio, tanto da paragonarsi ad una palma le cui radici sono ben salde nella terra. Il contrasto tra la piuma e l'albero è decisivo. L'esperienza dell'altrove ha reso 'volatile' la sua identità e il senso di appartenenza; solo il ritorno conferma la realtà fisica e concreta del mondo a cui appartiene chi si è assentato per un lungo soggiorno. L'esperienza del ritorno è di particolare interesse nel romanzo arabo di formazione e ha nel già citato Qindīl Umm Hāšsim un precedente non privo di interesse. Qui si narra di Ismā'îl, il terzo figlio di una famiglia egiziana che sacrifica tutti i suoi beni per fargli studiare oftalmologia in Inghilterra. Dopo sette anni di viaggio studio (si noti la coincidenza spaziale e temporale con il viaggio studio del narratore nel romanzo di Șāliḥ), Ismāi îl torna nel paese di origine per curare «un paese di ciechi». Come ha ben messo in evidenza Casini nella sua analisi del romanzo, la cecità è qui allegoria di un paese vissuto dal protagonista in totale stato di minorità nei confronti dell' $\mathrm{Eu}$ ropa, simbolizzata dal pensiero scientifico. Il romanzo è in realtà una critica all'atteggiamento di pedissequa imitazione dell'Occidente assunto in un primo momento dal protagonista, il cui scopo principale era quello di curare la 'cecità' del proprio paese personificato dalla cugina Fățima (Casini et al. 2013, 222). Tuttavia, dopo il suo ritorno nel quartiere di Sayyida Zaynab, Ismā îl vivrà una graduale trasformazione che gli permetterà di risolvere in una nuova originale sintesi quella dicotomia tra Egitto ed Europa, spiritualità e pensiero scientifico, che lo aveva portato alla follia e alla disgregazione della propria personalità. Oltre al messaggio veicolato dal racconto, considerato «uno dei grandi classici della narrativa araba moderna dedicati alla rappresentazione de "l'incontro culturale tra oriente e occidente" », un altro aspetto rilevante è l'impianto diegetico scelto da Yahyā Haqqī, il quale attira l'attenzione del lettore non tanto sulla vita che il protagonista conduce in Inghilterra quanto sulle conseguenze che tale soggiorno provoca sulla sua personalità.

«Passarono sette anni. La nave fece ritorno». I sette anni che Ismā ìl trascorre in Inghilterra sono racchiusi in questa frase. [...] La formazione di Ismā $َ 1$ in senso concreto, quindi, è già avvenuta in Europa, e il racconto si concentra su quanto accade dopo (Casini et al. 2013, 142). 
L'analisi di Paniconi potrebbe essere ugualmente applicata a La stagione della migrazione al Nord. Tuttavia, va osservato che, nonostante la somiglianza cronotopica tra le due opere, rispetto alla dicotomia tra Europa ed Egitto simbolizzata dalla follia passeggera del protagonista del racconto di Haqqī, nel romanzo di Saaliḥ si assiste ad un apparente ribaltamento totale di atteggiamento dei due personaggi principali rispetto al Nord e al Sud. Come si vedrà tra breve, il narratore, pur avendo anche lui vissuto sette anni in Inghilterra, rimane visceralmente legato al proprio villaggio di origine che rappresenta per lui l'unica fonte di serenità. Il ribaltamento è tuttavia apparente dal momento che nel romanzo di Șālih si instaura una dinamica di sdoppiamento e rispecchiamento tra narratore e protagonista. Questo accostamento tra due opere della narrativa araba che utilizzano strategie narrative del tutto simili per raccontare esperienze di viaggio altrettanto analoghe, sebbene veicolando messaggi diversi, mette bene in luce come la letteratura araba del Novecento abbia attribuito all'esperienza del ritorno una particolare funzione rigeneratrice di un'unità precedentemente perduta, di un'identità messa in crisi dall'altro e dall'altrove.

Ritorno dunque, come ritrovamento dell'identità dopo l'esperienza estraniante e alienante della migrazione. Una difesa dall'alienazione causata dal viaggio e dalla migrazione che in certi casi può provocare un profondo disagio psichico (Beneduce 1998), è il mantenimento del legame, anche solo psicologi$\mathrm{co}$, con la propria patria di origine pur stando in terra straniera.

Anche io ho vissuto con loro, ma ho vissuto con loro così, in superficie, senza amarli né odiarli. Avevo serbato questo piccolo villaggio nel mio cuore, lo vedevo con la fantasia ovunque mi voltassi [...]. Io, è sicuro, sono uno di quegli uccelli che vivono in una sola parte del mondo (Salih 2011, 67).

Nel romanzo di Sāliḥ, l'attitudine della voce narrante nei confronti dell'altrove non è partecipativa, ma da spettatore passivo degli eventi. Della sua vita privata verrà detto solo che è sposato con una donna probabilmente del suo stesso villaggio e che ha una figlia di nome Amal. Quando Wadd al-Rayyes, settantenne ancora aitante, amico del nonno del narratore, ammiccandogli gli dice: «dicono che le donne dei cristiani sono qualcosa al di là dell'immaginazione», egli risponde di non saperlo. «Ma che discorsi sono questi?», sbottò. «Un pezzo di giovine come te, nel fiore della gioventù, sta sette anni nel paese di Sodoma e Gomorra, e mi vieni a dire non lo so» (Salih 2011, 97).

Rapito dal ricordo del suo villaggio, è come se il narratore avesse messo tra parentesi i suoi sette anni spesi in Inghilterra. Gli odori di ogni acquazzone estivo a Londra lo riportano al villaggio natio, gli abitanti non li ha né amati né odiati. Niente ha cambiato la sua vita, se non il fatto di aver ottenuto un Dottorato in letteratura inglese che gli permetterà di ricoprire un posto al Ministero dell'Educazione a Khartum. Anche in questo caso, la cornice cronotopica che scandisce il prima e il dopo, il qui e l'altrove attraverso cui prende avvio la storia è costituita da un momento di contrazione e uno di dilatazione. «Nel paese di Sodoma e Gomorra» niente influisce sulla sua vita; il tempo e lo spazio sono sospesi. È solo qui nel 'nostro paese', nell'appartenenza al 'noi', che tutto acqui- 
sta un senso statico della vita. Il tempo è scandito dalle preghiere mattutine del nonno: «Era così da non so quanti anni, come una cosa fissa al centro dell'universo in movimento» (Salih 2011, 67). I rapporti uomo-donna sono sempre stati gli stessi dalla notte dei tempi: «Le chiacchiere che imparate a scuola da noi non servono. In questo paese gli uomini sono preposti alle donne» ${ }^{3}$, rinfaccia Wadd al-Rayyes al narratore quando gli chiede di convincere la vedova di Mușțafā a sposarlo. Il mondo del narratore ha una struttura in un certo qual modo tolemaica, dove al centro vi è il nonno, rappresentante di un mondo tradizionale fatto di preghiere e dinamiche sociali consolidate. All'universo tolemaico della voce narrante si contrappone quello di Mușțafā Sa īd.

\section{La ricerca di un'identità}

Come detto in precedenza, il romanzo ruota attorno alla domanda sulla vera identità di Muștafā Sa' ìd. Quando la voce narrante chiede ai suoi familiari di quest'uomo, gli viene risposto che era un sudanese venuto da fuori che si era stabilito nel villaggio cinque anni prima che il narratore tornasse. $\ll[\mathrm{A}]$ veva comprato un podere, costruito una casa e sposato una delle figlie di Mahmùd. Un uomo che si faceva i fatti suoi, non ne sapevano molto» (Salih 2011, 26). Muștafā racconterà la sua vera storia al narratore in una sorta di confessione, rivelandogli la propria identità. Come nelle Mille e una notte (opera i cui personaggi della storia cornice sono più volte evocati nel romanzo), si ha dunque una storia nella storia, tuttavia la cifra principale del romanzo di Ṣăliḥ è che il secondo grado del racconto si pone in relazione speculare con il primo. Inoltre, l'intreccio della narrazione fa sì che la confessione di Mușțafā si dilati e ritardi tramite prolessi e analessi che creano un effetto suspence. Come ebbe a scrivere Freud sull'Edipo re di Sofocle, la tragedia acquista tutto il suo interesse grazie al fatto che la rivelazione dell' 'identità di Edipo è «ritardata ad arte» dall'autore (Freud 1966, 243); il protagonista cioè, assassino e detective al tempo stesso, solo alla fine della storia scoprirà di aver ucciso il padre e sposato la madre, realizzando così il vaticinio dell'oracolo. Nel racconto della vita di Muștafā al narratore, emergono le tematiche classiche del romanzo arabo moderno: la colonizzazione inglese, i successi scolastici del giovane protagonista nelle scuole coloniali, le esperienze di studio prima al Cairo e poi a Londra. Il racconto segue un ordine apparentemente cronologico, in cui è possibile distinguere due fasi, l'infanzia fino al soggiorno in Egitto e la vita a Londra.

Appartenente ad una tribù, i Banū 'Ubayda, «la tribù che vive tra l'Egitto e il Sudan» e che durante l'invasione inglese ha tradito il proprio popolo e le proprie origini, Muștafāa è un uomo senza radici, e « $[\mathrm{g}]$ li uomini senza radici sono quelli che occuparono le cariche più alte al tempo degli inglesi» (Salih 2001, 72). Un uomo senza radici che lascia giovanissimo il proprio paese perché mosso dal «morbo della partenza». 
È inutile ingannare sé stessi. Quel lontano richiamo continua a rimbombarmi all'orecchio. Avevo creduto che vivere e sposarmi quil'avrebbero messo a tacere. Ma sono fatto così, o forse il mio destino è questo, qualunque ne sia il senso, non lo so. Razionalmente so ciò che si deve fare, cosa che ho messo in pratica in questo villaggio, con questo popolo di uomini felici. Ma oscure cose che ho nell'anima e nel sangue mi spingono verso zone lontane che mi appaiono e che è impossibile ignorare (Salih 2011, 84).

L'attrazione per l'altrove, per zone lontane, in un capovolgimento spaziale secondo cui 'l'altrove è il qui e qui è l'altrove', riecheggia il «via-di-qua » del racconto di Kafka La partenza, in cui, quando il servo chiede al proprio padrone che sta sellando il cavallo, dove sia diretto, questi gli risponde:

«Non lo so [...] purché sia via di qua, solo via di qua. Via di qua senza sosta, soltanto così potrò raggiungere la mia meta». «Dunque conosci la tua meta», osservò lui. «Sì», replicai, «l'ho detto, no? Via-di-qua ... ecco la mia meta» (Kafka 1985, 428-9).

Come ha notato Alfonso Iacono nell' incipit di un suo non più recente saggio, «Per il protagonista mettersi alle spalle il luogo della partenza diventa dunque esso stesso una meta $\gg$ (Iacono 2000, 9). Quando la migrazione diviene una fuga, la meta si identifica col via-di-qua kafkiano. Lo studio, i successi scolastici e accademici divengono così una via di fuga per Mușțafā Sa ìd, il cui obiettivo primario è anzitutto uscire dalla casa materna, in un periodo in cui i funzionari statali andavano per i villaggi in cerca di bambini da scolarizzare. Mentre i genitori nascondevano i figli in casa per timore che gli inglesi li rapissero, il piccolo Muṣtafā è per strada a giocare con altri bambini:

[...] giunse un uomo a cavallo in divisa che si fermò sopra di noi. I bambini fuggirono via e io rimasi a guardare il cavallo e l'uomo che gli stava sopra. $\mathrm{Mi}$ chiese come mi chiamassi e glielo dissi, mi chiese: «Quanti anni hai?», e gli risposi: «Non lo so». «Ti piacerebbe andare a scuola?», mi domandò. [...] «Potrò portare un turbante come questo?», domandai all'uomo indicando una cosa simile a una cupola sulla sua testa. L'uomo si mise a ridere e mi disse: «Questo non è un turbante, è un berretto, un cappello». [...] «Quando sarai grande ed uscirai dalla scuola e diventerai un funzionario dello stato, porterai un cappello come questo». «Andrò a scuola», gli dissi. [...] Andammo da un uomo con la barba, vestito di una giubba, il quale si alzò e dandomi dei buffetti sul capo mi disse: «Ma dov'è tuo padre?». Gli dissi che mio padre era morto e lui mi chiese: «Chi si prende cura di te?». «Voglio andare a scuola», dissi (Salih 2011, 42-3).

Dalle ultime battute di questa scena che ritrae Muṣțafā bambino affascinato dall'uomo a cavallo in divisa coloniale, si evince che il motivo per cui Mușțafā decide di andare a scuola non è tanto il desiderio di diventare un funzionario con in dosso il cappello di ordinanza del corpo inglese di stanza in Sudan, bensì il desiderio di lasciare le mura domestiche. Alla domanda dell'uomo con la bar- 
ba su chi si prendesse cura di lui, Mușțafā non risponde «la mamma», come ci si potrebbe aspettare da un bambino di otto anni. Un padre mai conosciuto e la freddezza di una madre inespressiva, incapace di accudimento e avara di amorevoli cure spingono Mușțafā ad andare 'via-di-qua'. L’amore non ricevuto dalla madre fa di Mușțafā «un bambino tormentato», incapace di lasciarsi andare, «di dimenticare il suo cervello». Pochi anni dopo, Elizabeth Robinson, la madre putativa di Muștafāa allorché lo accoglie come un figlio al Cairo insieme a suo marito Richard Robinson, si accorge di avere adottato un bambino speciale: «Tu, Mr. Sa'ìd, sei una persona completamente priva d'allegria», avrà modo di dirgli. Nella lettera che Elizabeth scriverà all'io narrante dopo la morte di Muștafāa descrive brevemente i tratti peculiari della personalità del suo Musy:

Sono occupata a scrivere un libro sulla nostra vita, di Ricky, Musy e me. Erano due grandi uomini, ciascuno a suo modo. La grandezza di Ricky stava nella sua capacità di trasmettere la felicità agli altri. Era felice nel vero senso della parola, la felicità si inondava da lui a tutti quelli con cui veniva in contatto. Musy aveva una mente geniale, ma era impulsivo. Era incapace di ricevere la felicità o di darla, tranne che a quelli che amava e che lo amavano veramente come Ricky e me (Salih 2011, 155).

La felicità del marito della signora Robinson si contrappone al triste Muștafā, che nel raccontarsi al narratore avrà modo di dire di essere stato un bambino incapace di amare ma che pretendeva amore dagli altri. Questo il contesto familiare e affettivo-emozionale entro cui collocare il capovolgimento spaziale tra il qui e l'altrove, capovolgimento raffigurato in un singolo luogo o spazio narrativo: la stanza segreta, rigorosamente chiusa a chiave, nella casa di Mușțafā Sa īd in Sudan. Questa stanza era «fatta di mattoni rossi, di forma oblunga e dalle finestre verdi. Il suo tetto non era piatto, come di norma, ma era convesso come il dorso di un toro» (Salih 2011, 34). È il luogo in cui Mușțafā serba tutti i suoi oggetti, taccuini, libri e foto della sua vita trascorsa in Inghilterra. La forma anomala della stanza con tetto spiovente e non a terrazza, come le altre case dei villaggi lungo il corso del Nilo, è la plastica evidenza di un'architettura all'europea. Ilibri in essa contenuti rappresentano l'enciclopedia' della cultura letteraria e scientifica europea, non vi è conservato «un solo libro in arabo», anche le copie del Corano sono traduzioni inglesi. Einstein, Freud, Wittgenstein, Kipling, Mann, Woolf, Smith, Keynes, sono solo alcuni degli autori dei libri conservati nella biblioteca privata di Muștafā. Questo luogo ha un alto valore simbolico a causa degli oggetti dai forti connotati emotivi che essi suscitano, nei quali è possibile trovare la risposta alla domanda sull'identità di Muștafā Sa ìd. La stanza, volendo usare un' immagine tolta alla cosmologia moderna, rappresenta una sorta di cunicolo spazio-temporale ${ }^{4}$, che mantiene Muștafā Sa ìd legato alla sua vita

4 Sui cunicoli temporali nello spaziotempo einsteiniano, si consiglia la piacevolissima lettura di Thorne $2019^{2}$ soprattutto: 503-518; l'autore ha ricevuto il premio Nobel per la fisica nel 2017. 
precedente, varcando la cui soglia si entra in un altro tempo e in un altro spazio: la Londra della sua giovinezza. La stanza in mattoni rossi di Mușțafā Sa īd è in negativo ciò che il villaggio sull'ansa del Nilo rappresenta per il narratore durante il suo soggiorno-studio a Londra. I due personaggi sono dunque l'uno il negativo dell'altro: il villaggio e la stanza in mattoni rossi sono due luoghi nel medesimo spazio che simbolizzano due mondi contrapposti, il Nord e il Sud, l'Europa e l'Africa, il Cristianesimo e l'Islam.

\section{II doppio}

Il rapporto speculare tra i due personaggi viene declinato attraverso una dinamica fatta di opposti e analogie che si dipanano lungo l'intera narrazione. Questi i tratti essenziali: il narratore è anonimo mentre il personaggio attorno a cui ruota la vicenda si chiama Mușțafā Sa ìd, vero e proprio protagonista. Muṣțafā è da subito associato ad uno spettro (tayf) che balugina beffardo agli occhi del narratore a più riprese e che si farà via via presente nelle varie fasi della storia. Entrambi hanno trascorso un lungo periodo di studio in Inghilterra dopo un notevole successo scolastico riscosso nel loro paese di origine. Il numero sette acquista un significato temporale che accomuna le due storie: sette anni è il tempo che il narratore ha trascorso in Inghilterra prima di tornare nel suo paese, sette sono gli anni di carcere a cui è stato condannato Mușțafā Sa ìid; sette sono i mesi di assenza del narratore dal villaggio, durante i quali la vedova di Muṣṭafā uccide il secondo marito prima di suicidarsi. Il narratore ha un'esperienza di sdoppiamento nello specchio della stanza segreta di Mușțafā. Ultimo tratto accomunante i due personaggi è il rapporto con le acque del Nilo.

Questi elementi vanno a costituire un tema centrale di tutto il romanzo, a cui la letteratura critica del secolo scorso, salvo poche eccezioni (Siddiq 1978, 85-7), ha prestato poca attenzione, ossia il tema del doppio, che nella letteratura romantica ha assunto una grande importanza e che è stato oggetto di svariate interpretazioni. Qui, a causa delle suggestioni che la storia stessa evoca, faremo principalmente riferimento al saggio di Otto Rank, Il doppio, uno studio psicoanalitico, edito dapprima nella rivista della Società Psicoanalitica di Vienna Imago (1914) e successivamente in un volume a parte nel 1925. Tra i numerosi esempi di doppio letterario analizzati da Rank, uno sembra di particolare interesse per il lettore del romanzo di Șāliḥ, ovvero quello tratto da William Wilson, la ben nota novella di Edgar Allan Poe. Qui si racconta di due compagni di scuola che sono l'uno il sosia dell'altro, tuttavia con il procedere della storia, il sosia si trasforma prima in un rivale poi in una vera e propria presenza persecutoria per il protagonista. Durante un ballo in maschera, il protagonista, riconosciuto il suo sosia, lo sfida a duello e lo trafigge con la spada. Segue la scena del rispecchiamento della propria immagine che viene confusa con quella del proprio alter ego, il quale così si rivolge al protagonista: «Tu vivevi in me, ora che io muoio, puoi vedere in me la tua immagine: uccidendomi ti sei ucciso» (cfr. Rank 2001, 38-9). 
Il brano ha una certa risonanza con il racconto dell' io narrante nel romanzo di Șāliḥ. Quando egli entra per la prima volta nella stanza segreta di cui Mușțafā gli aveva lasciato la chiave, ha un'esperienza del tutto simile.

Accesi un fiammifero. La luce fu per i miei occhi come un'esplosione. E dal buio usci un viso torvo dalle labbra serrate che conoscevo ma non ricordavo più. Avanzai verso di lui livido. Era il mio nemico, Mustafà Sa’̀̀d. Al viso si aggiunse un collo, e al collo due spalle e un petto, poi un busto e due gambe. E mi trovai in piedi davanti a me stesso faccia a faccia. Questo non è Mustafà Sa'ìd. È la mia immagine che mi guarda torva in faccia dallo specchio (Salih 2011, 144).

L'altro come doppio di sé, la cui immagine riflessa nello specchio si confonde con la propria. Perché il doppio? Qual è il suo significato? L'interpretazione che ne offre Rank adotta la metapsicologia freudiana come quadro epistemologico che negli stessi anni in cui Rank scriveva si andava costituendo, soprattutto per quanto riguarda la teorizzazione del narcisismo e del suo rapporto con la morte $^{5}$. Secondo l'interpretazione di Rank, si tratta dello stesso personaggio diviso in due a causa della presenza nella stessa persona di due aspetti contrastanti del carattere ${ }^{6}$. La contrapposizione è tale da causare lo sdoppiamento dell' Io in due personaggi diversi tramite meccanismi di scissione e proiezione a scopo difensivo. Nella nostra storia, il narratore aderisce al mondo rurale e contadino del paese di origine, da cui l'insistenza sul tema delle origini e dell'identità originaria che, rappresentando un punto di riferimento in un «universo in movimento», è fonte di sicurezza e serenità. L'altro personaggio, Mușțafā Sa î̀d, è il frutto della «missione civilizzatrice in Africa», un uomo educato alla occidentale che ha rapporti con donne inglesi. La differenza con gli altri personaggi dei romanzi della prima generazione della Nahda araba, come il letterato in Adìb e Ismā îl in Qindīl Umm Hãšsim, oltre al differente uso dei tropi letterari e dei messaggi da questi veicolati, sta nello scavo psicologico che l'autore opera nei confronti del protagonista. La descrizione dell'infanzia di Muștafā, il suo rapporto con le donne inglesi e con il paese di origine, assumono non più una dimensione metaforica di antitesi tra città e campagna, arretratezza e modernità e, in ultima analisi, mondo arabo ed Europa, ma permettono di scendere nella profondità del soggetto, le cui contraddizioni interne danno vita a due personaggi distinti ma speculari.

Muștafā Sa ìd muore durante una inondazione del Nilo. La morte del protagonista non lo annichila ma lo presenzia ancor più fortemente nella mente $\mathrm{e}$ nell'immaginario del narratore, come uno spettro il cui nome tornerà più e più volte come una condanna e una persecuzione. È interessante a questo proposi-

5 Si noti a questo proposito che il saggio di Freud Introduzione al narcisismo sarà pubblicato lo stesso anno in cui Rank dà alle stampe sulla rivista Imago il suo studio sul doppio.

6 Sulla divisione dell'io e la personalità cosiddetta 'schizoide', i classici di riferimento sono Laing 1969 e Sass 2013, il quale pone in parallelo la sua esperienza psichiatrica con la produzione letteraria e il pensiero filosofico europeo di stampo modernista. 
to osservare la strategia narrativa che l'autore mette in atto, utilizzando ciò che Freud chiama «negazione» (Verneinung) come difesa dal ritorno del rimosso, che non fa che confermarne la presenza. In altre parole, « $\ll i]$ l contenuto rimosso di una rappresentazione o di un pensiero può dunque penetrare nella coscienza a condizione di lasciarsi negare» (Freud 1978, 198). Almeno in due punti distinti del romanzo si fa riferimento a Muștafā Sa îd come ossessione persecutoria tramite una negazione del fenomeno. Ė il caso dell' incipit del IV capitolo:

Comunque, signori miei, spero non abbiate l'impressione che Mutafà Sa'ìd fosse divenuto un'allucinazione che mi ossessionava nella sosta e nel cammino. A volte potevano passare dei mesi senza che mi venisse in mente. Ad ogni modo era morto, annegato o suicida, Dio solo lo sa. Migliaia di persone muoiono ogni giorno e se ci soffermassimo a disquisire sul perché e su come ognuna di esse sia morta, che ne sarebbe di noi vivi? (Salih 2011, 79).

Si noti che la traduzione «non abbiate l'impressione che» rende l'espressione araba là yatabādaru 'ilā 'a dhānikum che letteralmente potrebbe essere reso con «non si presenti subito alla vostra mente». È come se il narratore, rivolgendosi direttamente ai suoi ascoltatori, volesse negare qualcosa in realtà di ineluttabile; nega per asserire. Il resto della storia non fa che confermare tale ineluttabilità. D'altronde il tempo in cui l'io narrante non pensa o non è sotto l'influenza del suo doppio è pressoché inesistente per il lettore, fa parte del non-narrato. Lauspicio che nel brano testé citato formula il narratore nei confronti dei suoi interlocutori immaginari è lo stesso che Muștafă scrive nel suo testamento al protagonista-narratore riferendosi ai suoi stessi figli.

Non so cosa penseranno di me allora; forse proveranno compassione, oppure mi trasformeranno nella loro fantasia in un eroe. Questo non importa, l'importante è che la mia vita non giunga dall'ignoto come uno spirito maligno che arrechi loro danno (Salih 2011, 84).

Non sapremo niente della vita dei figli di Mușțafā Sa ìd, ma è plausibile pensare che si verificherà proprio ciò che il padre scongiura per loro.

Dopo la morte del protagonista è come se il tempo e lo spazio si dilatassero. Niente di particolare avviene nella vita del narratore, gli unici punti salienti sono i ricordi e le persone che del tutto involontariamente gli riportano alla memoria il fatidico personaggio. Tutto comincia «due anni dopo» la morte del protagonista: passeggeri incontrati per caso su un treno per Khartum che dichiarano di essere stati compagni di scuola di Mușțafā Sa ì id, colleghi e vecchi compagni di studi che affermano di sapere chi fosse il primo sudanese ad aver sposato una donna inglese. Ciò nonostante, la voce narrante dichiara di aver vissuto per ben venticinque anni senza averlo conosciuto, un periodo della vita di cui non viene detto quasi niente. In ultima analisi, sembra di poter concludere che senza una qualche presenza di Mușțafā Sa ìd la vita dell'io narrante è priva di senso.

Avevo vissuto venticinque anni senza sentir parlare di lui né vederlo, poi così all'improvviso me lo trovo davanti in un luogo dove non ha simili, ed ecco 
Mușțafā Sa īid, mio malgrado, farsi parte del mio mondo, idea nella mia mente, spettro che non vuole andarsene per la sua strada. [...] Anche a Khartum si presentò lo spettro di Muștafā Sa î̉, $[\ldots]$ come fosse un genio uscito fuori dalla sua prigione, che avrebbe continuato a bisbigliare agli orecchi degli uomini, ma per dire cosa? Non lo so (Salih 2011, 68 e 73).

Una vita normale di un brillante studente sudanese che emigra per seguire il corso della propria formazione viene sconvolta da una presenza che segnerà per sempre la sua vita. I venticinque anni si oppongono a quell'espressione avverbiale di tempo, «così all'improvviso» (hakad $\bar{a}$ fağ atan), suo malgrado, indipendentemente dalla sua volontà, lo spettro gli si para dinanzi. Questo brano si configura come una sorta di palinsesto letterario, un ipertesto che richiama al lettore arabo i testi fondativi della sua 'enciclopedia'. Nella Storia del pescatore e del demone raccontata da Shahrazād nelle Mille e una notte, si narra la storia di un demone che era stato imprigionato in un vaso di rame per mille e ottocento anni. Liberato da un pescatore che recupera l'anfora dal fondo del mare, decide di uccidere il suo liberatore senza offrirgli una via di scampo. Si aggiunga inoltre che il tema del demone persecutore che sussurra malvagità alle orecchie degli uomini è tramandato da una delle più antiche sure del Corano $(114,5)$. Come si nota lo spettro di Muṣțafā Sa îd si inserisce in una tradizione molto antica. Qual è dunque il significato del genio equiparato a Satana all'interno del romanzo?

Nel saggio Das Unheimliche (1919), Freud prende in analisi i vari temi letterari che creano nel lettore-spettatore l'effetto di 'perturbante', di 'non-familiare', come l'etimo della parola tedesca indica. Tra questi spicca il tema del doppio. Ciò che accomuna tutti i temi analizzati dal padre della psicoanalisi, dall'animismo all'onnipotenza dei pensieri, dal rapporto con la morte alla ripetizione involontaria e il complesso di evirazione, vi è il già evocato concetto di 'ritorno del rimosso', intrinsecamente connesso con la teoria freudiana della rimozione. Secondo Shelling, l'Unheimlich «è tutto ciò che avrebbe dovuto rimanere segreto, nascosto, e che è invece affiorato» (Freud 1977, 86). Il profondo turbamento che lo spettro suscita in chi lo vede è dovuto al fatto che il contenuto rimosso che esso simbolizza si palesa all'improvviso, in un momento inaspettato, o meglio, nel momento meno opportuno. «Poi così all'improvviso me lo trovo in un luogo dove non ha simili», in questa frase del narratore si nota un' indicazione di spazio («luogo dove non ha simili») e di tempo («all'improvviso») che sconcertano l'io narrante proprio a causa del suo manifestarsi malgrado la propria volontà.

In un'altra interpretazione che Rank offre nel suo studio sul doppio, il sosia avrebbe una funzione di perpetuazione dell'Io come reazione e difesa dalla paura della morte. In ultima analisi, il sosia non sarebbe altri che la personificazione di un narcisistico desiderio di immortalità.

La frequente eliminazione del proprio doppio, attraverso cui il protagonista cerca una definitiva salvezza dalle persecuzioni del suo io, è in realtà un suicidio reso indolore dal fatto che è un altro io ad essere ucciso. Si è qui in presenza dell'inconscia illusione di separarsi da un io malvagio e che merita di venir punito, condizione d'altronde che sembra esser la premessa di ogni suicidio (Rank 2001, 99). 
È dunque possibile concludere che il doppio per Rank altri non è che l'espressione metaforica del ritorno del rimosso del protagonista, il quale, non potendosi liberare del proprio passato, lo personifica in un alter ego persecutorio, la cui morte prelude al suicidio del protagonista. La morte del doppio e successivamente il suicidio assumerebbero un valore liberatorio dalla paura della morte (Rank 2001, 97). Narcisismo, morte e doppio si trovano dunque in una relazione intrinsecamente interconnessa. Nei racconti in cui il protagonista è affetto da narcisismo, lo specchio e il rispecchiarsi rivestono un ruolo centrale. Per Muștafā Sa īd lo specchio è strumento di moltiplicazione e quindi di perpetuazione della propria immagine; come si dirà a breve, l'elemento femminile, che pure nello specchio si riflette insieme a Mușțafā, è tuttavia in una posizione del tutto strumentale, al servizio dell'ego del protagonista.

\section{II protagonista e il femminile}

Nei primi romanzi della Nahḍ araba in cui il viaggio di formazione ha un ruolo centrale nella struttura narrativa, il protagonista intesse spesso una relazione d'amore con una donna europea. La relazione diviene spesso metafora della totale identificazione del protagonista con l'Europa, a cui segue una crisi identitaria profonda. Nel romanzo La stagione della migrazione al Nord, il personaggio Muștafā Sa īd vive le sue avventure con donne incontrate a Londra in modo asettico, cinico, fino a rasentare la crudeltà. Una crudeltà simbolizzata dal sadismo del chirurgo che opera con estremo distacco sul suo paziente. La camera da letto in cui porta le «femmine» conquistate nel gioco seduttivo sempre paragonato ad una caccia, è descritta ora come un cimitero, ora come un campo di battaglia ora come una camera operatoria.

Nel mio cuore non c'era una stilla d'allegria [...]. La mia stanza da letto era una sorgente di tristezza, il germe di un male assassino. L'infezione le aveva contagiate mille anni fa, ma io avevo riattizzato i focolai del morbo perché riesplodesse e uccidesse. [...] Entrò nella mia stanza da letto vergine candida $\mathrm{e}$ ne uscì portando nel sangue il germe del male. Morì senza pronunziar verbo. La mia galleria d'esempi era inesauribile (Salih 2011, 51 e 55).

Ann Hammond, Sheila Greenwood, Isabella Seymour e infine Jeane Morris sono le donne nominate durante il processo in cui Muștafā è imputato di omicidio plurimo. Eros e thanatos, amore narcisistico e amore distruttivo, sadismo e masochismo sono le diadi che rappresentano la cifra dei rapporti intrattenuti da Muștafā con l'altro femminile durante la sua vita a Londra. Tutte le donne nominate, eccetto Jean Morris che diventerà sua moglie, subiranno un destino di morte, per scelta o per condizione. Come dichiarato dallo stesso protagonista, il germe del desiderio di morte infetta le sue vittime, il suo non è un amore generativo ma distruttivo.

Durante il processo per le morti di cui è considerato responsabile, gli viene rinfacciata la sua totale incapacità di amare. 
Lei, Mr. Said [...] malgrado la sua levatura scientifica, è uno stolto. Nella sua formazione spirituale c'è una zona d'ombra, per questo ha sciupato la più nobile capacità concessa da Dio agli uomini: la capacità d'amare (Salih 2011, 72) 7 .

L'amore narcisistico di origine infantile del protagonista è più volte evocato nella storia che Muṣțafā racconta al narratore.

Una realtà della mia vita era questa: come il caso abbia per me riservato una moltitudine di persone che mi hanno aiutato e preso per mano, una moltitudine verso cui non provavo nessun senso di riconoscenza. Ne accettavo l'aiuto come fosse un dovere che assolvevano nei miei confronti. [...] Avevo quindici anni, ma chi mi vedeva credeva che ne avessi venti, così padrone di me stesso, come un otre gonfio: dietro di me c'era la storia di un sensazionale successo scolastico, le mie armi erano tutte in quell'affilato coltello che avevo nel cranio, mentre nel petto avevo un freddo, arido sentimento, come se la sua cavità fosse stata riempita di pietre (Salih 2011, 44 e 48).

Ricordando la lezione di Bachtin, poiché tra mondo raffigurante e mondo raffigurato c'è sempre una sottile impercettibile relazione, non è difficile per il lettore intravedere le ragioni di tanto amor di sé. Il piccolo Mușțafā, orfano di padre, lascia la madre per seguire il suo destino. L'addio tra i due è caratterizzato dalle fredde parole che la madre rivolge al figlio. Non un'emozione trapela dal volto di lei, che viene paragonato a quello di una maschera. Le prime pulsioni sessuali il dodicenne Muṣtafā le proverà per la signora Robinson al momento del loro primo incontro alla stazione dei treni al Cairo:

D'un tratto sentii le braccia della donna cingermi il collo e le sue labbra sulla mia guancia. In quel momento, in piedi lì sulla banchina della stazione, in mezzo a un carosello di suoni e sensazioni, con le braccia della donna avvolte intorno al collo, la sua bocca sulla mia guancia, l'odore del suo corpo, uno strano odore europeo che mi solleticava il naso, il suo petto che sfiorava il mio, sentii, io, bambino di neanche dodici anni, un imprecisabile desiderio ${ }^{8}$ sessuale che non avevo mai conosciuto in vita mia, ed ebbil'impressione che Il Cairo, quel grande monte a cui mi aveva portato il mio cammello, fosse una donna europea... (Salih 2011, 46).

Ciò che la madre naturale non ha saputo essere per Muṣṭafā, viene personificato dalla madre adottiva, che non abbandonerà il figlio putativo anche nei momenti più difficili. Il primo incontro con l'alterità femminile, vissuto alla stazione

7 Si noti che in questo passo la traduzione italiana di Francesco Leggio rende la parola țāqa con «possibilità», mentre qui si ritiene più appropriato tradurre con «capacità ». Il protagonista non è infatti capace di provare amore se non per sé stesso.

8 Nel testo originale il termine è šahwa, che Leggio rende con «impulso», termine tecnico pertinente al linguaggio della psicoanalisi, derivato da «pulsione» (Freud usa il termine Trieb, in arabo gariza) su cui è basato il funzionamento del modello psicoanalitico freudiano, detto per questo anche «modello pulsionale» 0 «teoria delle pulsioni» (cfr. Freud 1976, 13-35). Qui tuttavia è di desiderio che si parla, ovvero la pulsione è personificata e percepita dal piccolo Muṣțafā come primo anelito verso l’altro-da-sé femminile. 
del Cairo, prefigurerà il futuro della vita sessuale e affettiva di Mușțafā. Giunto a Londra, il successo accademico-professionale arride al giovane protagonista. Prodigiosa intelligenza unita a incapacità di lasciarsi coinvolgere dalle donne che incontra fanno dell'uomo Muṣtafā un implacabile cacciatore di prede femminili. Carico di fascino per la sua abilità, cultura e soprattutto per le sue origini esotiche, non avrà difficoltà ad attirare nel suo appartamento giovani donne inglesi in cerca di avventura, di un diversivo o di trasgressione in una società ancora sin troppo vittoriana. La camera da letto era l'obiettivo della «caccia»; adibita a questo scopo, era curata in ogni minimo dettaglio: incensi, candele, piume di struzzo e tappeti orientali ricreavano un'atmosfera esotica colma di fascinazione. Ma soprattutto i muri della camera da letto erano coperti di specchi «sicché quando andavo a letto con una donna avevo l'impressione di andare a letto con un intero harem contemporaneamente» (Salih 2011, 52). L'orgiastico riflesso della propria immagine unita a quella di infinite altre donne è la plastica rappresentazione del narcisismo del protagonista. Le figure femminili e la loro storia sono un accessorio dell'atto predatorio. Una sola donna riuscirà a coinvolgerlo in una tormentata relazione sentimentale. Aveva conosciuto Jean Morris in una delle feste in casa a cui era solito partecipare, ma lei non si era lasciata conquistare facilmente. «Tutto ciò che avvenne prima di incontrarla fu un preludio e tutto ciò che feci dopo averla uccisa fu un atto di dolore, non per averla uccisa, ma per la menzogna della mia vita» (Salih 2011, 50). Una delle linee guida interpretative di questa analisi vuole leggere gli amori sofferti di Muṣțafā alla luce di alcuni concetti della psicoanalisi classica. Ma a differenza di altre letture che hanno applicato quasi meccanicamente il prisma freudiano al testo di Șâlih (John, Tarawneh 1988) e di molta letteratura critica che ha posto l'accento sul significato metaforico che la misoginia di Mușțafā assumerebbe in un'ottica tutta post-coloniale (Greesey 1997; Hassan 2003; Saeed Adam 2015), qui si è preferito assumere il problema del soggetto risvegliato nel lettore dai personaggi letterari, privilegiando una lettura per così dire 'in prima persona', piuttosto che una visione del personaggio come metafora di un prodotto storico-culturale.

In un percorso a ritroso, sembra che Jean ed Elizabeth rappresentino per Mușțafā, sebbene in forma diversa, dei sostituti della figura materna. Spinto da forze impalpabili lontano dalla terra di origine (anch'essa simbolo materno), ricerca quel contatto mai avuto con la madre reale nell'altrove-qua', tramite quel processo di sostituzione che Freud ha ben descritto' ${ }^{9}$ che fa sì che l'altro si trasformi nella persona più familiare e che anche a Londra il protagonista possa ritrovare quella madre dalla maschera impenetrabile, tanto desiderata ma irraggiungibile. Da qui ha origine il narcisismo del personaggio. Il bambino, non sentitosi amato dalla madre naturale, avrebbe ritirato la propria libido su sé stesso, fissando così il proprio carattere narcisista che gli permetterà di mante-

9 «Non senza ragione il lattante attaccato al petto della madre è diventato il modello di ogni rapporto amoroso. Il rinvenimento dell'oggetto è propriamente un ritrovamento» (Freud 1975, 107-8), corsivo mio. 
nersi in vita, a prezzo non solo di non poter instaurare fecondi rapporti amorosi con le donne che incontra, ma soprattutto di nutrire un rancore ancestrale e un desiderio di vendetta verso la madre stessa. Ann Hammond, studentessa di lingue orientali a Oxford, Sheila Greenwood, cameriera in un ristorante di Soho, Isabella Seymour, moglie di un chirurgo di successo e madre di famiglia, si invaghiscono tutte di un uomo che racconta loro seduttive storie esotiche con il solo spirito di conquista predatoria. La giovane Ann Hammond si era più volte dichiarata schiava del suo amato, in un gioco d'amore fatto di recite e di gesti teatrali dal sapore orientaleggiante: incensi, tuniche, cuscini di piume di struzzo e poesie arabe antiche adornavano il loro 'palcoscenico'. Prima di suicidarsi col gas gli dedicherà un messaggio di maledizione, probabilmente per un amore non ricambiato. Durante una conferenza allo Speakers' Corner di Hyde Park, Mușṭafā si avvicina a Isabella Seymour sino a sentirne il calore e l’odore. «Sentii l'odore del suo corpo, quello stesso odore con cui mi aveva accolto la signora Robinson sulla banchina della stazione del Cairo». Ad invito accettato, i due cominciano a frequentarsi per un mese. Lui le dice di chiamarsi Amīn Hasan, di essere orfano dei genitori entrambi annegati nel Nilo; lei vedeva in lui «un simbolo, non una realtà »; dietro una sua fotografia gli scriverà la dedica: «Tua fino alla morte. Isabella». Infine Husnā Bint Maḥmūd, la moglie che Muṣṭafā Sa i ìd sposerà dopo essere tornato in Sudan e che gli darà due figli, ammette che il loro rapporto non era un rapporto di amore.

«Hai amato Mustafà Saìd?».

$[\ldots]$

«Era un padre per i miei figli».

Se la mia impressione era giusta, la voce non era triste, ma vi era un gemito. La lasciai ai sussurri del silenzio, forse avrebbe detto qualcosa. Si, eccola:

«È stato un buon marito e un buon padre. Finché è vissuto non ci ha fatto mancare niente».

Husnā fa parte dell'ultimo anello di quella catena di morte che travolge le donne che si sono unite a Muștafā. Costretta a sposarsi a Wadd al-Rayyes, dopo averlo evirato lo uccide prima di suicidarsi. Morte e suicidio, questo il fil rouge che corona tutte le storie che Mușțafā intesse con le donne che incontra. Per scelta o per destino inesorabile, la morte delle donne di Muṣțafā sembra un effetto del loro rapporto con un arabo-africano dalla storia avvolta nel mistero. Ciò che sembra emergere dal testo dunque, non è tanto un desiderio di vendetta del protagonista nei confronti di un intero popolo o nazione, quanto piuttosto un rancore atavico nei confronti della madre. «Il mondo improvvisamente si era capovolto da cima a fondo. L'amore? L'amore non fa questo. È il rancore. Io sono pieno di rancore e chiedo vendetta $\gg$ (Salih 2011, 143). L'universo statico del narratore si è rotto, quell' idillio fatto di certezze infranto, anche nel villaggio una donna può uccidere un uomo perché non vuole sottostare a leggi ancestrali. Gli uomini non sono più preposti alle donne; donne inglesi e donne africane possono essere autrici del proprio destino, ma non può che essere un destino di morte. Jean Morris, la donna che Muṣțafā Sa īd sposa, instaura con lui un rap- 
porto sado-masochista, dove il protagonista, da cacciatore diviene preda, vittima passiva dei capricci autodistruttivi della moglie. «Non era odio. Era amore incapace di esprimersi. L'amavo in maniera contorta. E anche lei». Ciò che più di un secolo fa Freud ascriveva alla sessualità femminile, sembra attagliarsi molto bene a entrambi i personaggi:

A rigore queste donne amano, con intensità paragonabile a quella con cui sono amate dagli uomini, soltanto sé stesse. In verità i loro bisogni non le inducono ad amare, ma piuttosto ad essere amate; e si compiacciono degli uomini che soddisfano questa loro esigenza (Freud 1975, 459).

Lo scopo di Jean era quello di essere amata: «Ma era di un'intelligenza e di un'amabilità eccezionale, quando voleva, circondata ovunque fosse da uno stuolo d'ammiratori svolazzanti attorno a lei come mosche» (Salih 2011, 162). Il narcisismo autodistruttivo di Jean trova il suo compimento nella sua relazione con Mușțafā. Jean gli infliggerà lunghe e forzate astinenze sessuali, l'unico momento in cui avranno un rapporto sarà in un parco pubblico, dimentichi di tutto e di tutti. All'estasi dei rari amplessi si alternano scontri e torture fisiche e psicologiche. Lei gioca a far la seduttrice con uomini incontrati per strada o al ristorante, oggetti di altri uomini erano facilmente rinvenibili nel loro appartamento. Tutto conduce verso un destino inesorabile. Jean provoca l'Otello che è in Mușțafā. «"Che cosa t'impedisce di uccidermi? Che aspetti? Forse aspetti di trovare un uomo sopra di me. E pure allora non penso che farai nulla. Ti siederai sul letto e piangerai" $\gg($ Salih 2011, 168). È grazie alle umiliazioni che Jean gli infligge che il protagonista potrà finalmente piangere al ricordo della notizia che gli annunciava la morte della madre. Quando Jean lo sfida con tono «non privo di dolcezza», dicendogli, «Tu, amore mio, non sei tipo da uccidere», suscita nel protagonista il ricordo delle ultime parole che la madre gli rivolge prima della sua partenza: «È la tua vita e sei libero di disporne». Jean, sostituto della madre, non è che il ritorno del rimosso di Muștafā. «Nei suoi occhi c'era una sfida e un richiamo che suscitò lontane passioni nel mio cuore». Laddove la signora Robinson personifica la madre agognata e mai avuta, Jean risveglia nel protagonista il complesso della «madre morta $\gg(\text { Green } 2018)^{10}$. Afflitta dal lutto del marito, la madre non è più capace di dedicare al figlio le attenzioni e le cure che il bambino le richiederebbe. Matura così in Mușțafā una profonda depressione che sfocerà in una totale incapacità di amare le donne che incontra. L'unico momento in cui Muștafā sentirà finalmente di amare è quando esaudirà il desiderio di morte di Jean. L'omicidio si consuma tramite un coltello che

10 Il complesso della «madre morta», elaborato dallo psicoanalista francese André Green, sembra attagliarsi bene alla descrizione del problema del soggetto rievocato dal protagonista del romanzo di Șālih. Esso richiama non tanto ad una reale perdita della madre, quanto piuttosto alla perdita delle attenzioni materne dal momento che è la madre stessa a subire un lutto. Il bambino, non più al centro dell'universo materno, cade in depressione. «Il tratto essenziale di questa depressione è che essa si determina in presenza dell'oggetto, lui stesso assorbito in un lutto» (Green 2018, 228). 
lentamente le affonda tra i seni. La penetrazione mortale si oppone a quella genitale generatrice di vita. In quel momento «l'universo col suo passato, il suo presente e il suo futuro si condensò in un solo punto prima e dopo il quale non era nulla». Al mondo tolemaico del narratore si oppone l'universo in contrazione di Mușțafā Sa î̀. Il momento apicale non sarà più rappresentato dall’amplesso con la propria preda di caccia, ma dall'uccisone di quella donna che più lo ha fatto soffrire, la sola che lo ha fatto amare, quella «madre morta» che lo implora di consumare finalmente la sua vendetta.

\section{Conclusioni}

All'inizio di questa analisi del romanzo più noto di al-Ṭayyib Șāliḥ, è stato rievocato l'ultimo saggio del fisico teorico Carlo Rovelli Helgoland. Qui l'autore ricostruisce per un pubblico di non specialisti, la storia di quella branca della fisica moderna nota come meccanica quantistica. Helgoland è il nome dell' isola in cui il giovane scienziato Werner Heisenberg, ritiratosi in cerca di salute e concentrazione, formulerà il ben noto principio di indeterminazione che è ancora oggi uno dei fondamenti della fisica dei quanti. Una delle interpretazioni che della meccanica quantistica offre Rovelli è che su scala subatomica la descrizione delle leggi di natura in terza persona, come avviene per la meccanica classica, non è più possibile. È solo all'interno di un'ottica relazionale tra oggetti o siste$\mathrm{mi}$ fisici che è possibile immaginare le sconcertanti leggi del mondo quantistico.

Insomma - conclude Rovelli - anche se sappiamo tutto quello che c'è da sapere in una situazione particolare su un oggetto singolo, se questo oggetto ha interagito con altri non sappiamo tutto di lui: ignoriamo le sue correlazioni con gli altri oggetti dell'universo. La relazione tra due oggetti non è qualcosa che sia contenuta nell'uno e nell'altro: è di più (Rovelli 2020, 103).

Questa conclusione si sposa felicemente con conclusioni del tutto simili raggiunte in altre discipline, come la psicologia o la psicoanalisi. Si pensi al ben noto principio gestaltista secondo cui l'intero non è dato dalla semplice somma delle singole parti che lo compongono. Similmente in psicoanalisi Thomas Ogden ha posto l'accento sul concetto di terzo analitico intersoggettivo: all'interno della dinamica psicoanalitica la relazione tra chi parla e chi ascolta crea un 'campo analitico' che va oltre le caratteristiche dei singoli attori della relazione analitica presi separatamente (Ogden 1994; 1999; 2013, 46-9). Credo che queste considerazioni debbano a fortiori essere tenute in considerazione anche in letteratura e in critica letteraria. La lettura post-coloniale de $\mathrm{La}$ stagione della migrazione al Nord coglie un elemento senz'altro essenziale del romanzo. L'esperienza dei due personaggi a Londra, i molteplici riferimenti all'invasione inglese in Sudan non lasciano dubbi, ma come evocato nell' introduzione, il contesto storico-politico è il quadro entro cui collocare l'intera storia ma non esaurisce tutto il significato delle relazioni che il protagonista intesse con il mondo femminile. Non solo perché anche nella figura della moglie sudanese si ripropone la medesima dinamica di morte, ma è soprattutto 
nella tormentata storia matrimoniale tra Mușțafā e Jean che il prisma postcoloniale non tiene conto delle dinamiche profonde che si instaurano tra $\mathrm{i}$ due personaggi. Piuttosto quindi che pensare a un conquistatore africano che si vendica su donne inglesi descritte come metafora di un paese occupante, qui si è preferito porre l'accento sulle dinamiche di identificazione, sdoppiamento e proiezione che entrano in gioco nella complessa relazione tra testo, protagonista/i, lettore (Holland 1986).

Al tragico destino del protagonista, risucchiato dalle acque del Nilo durante un'alluvione, si oppone quello colmo di speranza del suo alter ego, la voce narrante, che alla fine del romanzo decide di gettarsi nelle acque del Nilo per raggiungere la riva Nord. Il personaggio-narratore, che nella vita è sempre stato aggrappato al suo paese di origine (la madre) e che non ha mai potuto scegliere niente a causa della presenza troppo ingombrante di uno spettro persecutorio, sceglie di tuffarsi e nuotare verso Nord per tornare là dove aveva vissuto per sette anni. In un momento di crisi suicida, sceglie di rimanere in vita; tuttavia, questo atto di rinascita dalle acque sorgenti di vita è scandito da un attimo di grottesca ilarità. Egli grida aiuto «come un attore comico che strilla in un teatro»; non solo non è un grido credibile, ma sicuramente nessuno lo prenderà sul serio. L'epilogo sembra essere la metafora del soggetto che da Sud vuole migrare al Nord, un Nord freddo, incapace di udire le grida di aiuto di chi vuole raggiungere le sue rive. Epilogo sino troppo evocativo di una realtà che sembra ripetersi ancora oggi. Un grido di aiuto tuttavia, che non chiede solo salva la vita, ma racchiude in sé una richiesta più profonda, il ritrovamento di quell'unità intima del soggetto persa durante l'esperienza migratoria. L'elemento del doppio sintetizzato nell'unico personaggio narratore-Mușțafā è l'immagine di questa intima contraddizione tra attività e passività, forze vitali e autodistruttive, pulsione di vita e pulsione di morte insite in ogni essere umano. Ecco forse il motivo del successo di un classico che ancora oggi non cessa di parlarci.

\section{Riferimenti bibliografici}

Amyuni, M.T. 1998. “Ṣāliḥ, al-Ṭayyib (1929-)” Encyclopedia of Arabic Literature, a cura di J.S. Meisami, e P. Starkey, II, 680-1. London-New York: Routledge.

Bachtin, M. 1979 (1975). Estetica e romanzo. Torino: Einaudi.

Bakkār, T. 2015. al-T̄ābit wa-l-mutahawwil, prefazione a Șāliḥ T., Mawsim al-hiğra ilā šimāl, Dār al-ğanūb li-l-našr, Tūnis: 9-28.

Beneduce, R. 1998. Frontiere dell'identità e della memoria: etnopsichiatria e migrazioni in un mondo creolo. Milano: Franco Angeli.

Benslama, F. 2002. La psychanalyse à l'épreuve de l'islam. Paris: Flammarion.

Casini L. et al. 2013. Modernità arabe. Nazione, narrazione e nuovi soggetti nel romanzo egiziano. Messina: Mesogea.

Eco, U. 2016 (1980). I limiti dell'interpretazione. Milano: La Nave di Teseo.

Freud, S. 1966 (1899). L'interpretazione dei sogni. In Opere di Sigmund Freud, III, Torino: Bollati Boringhieri.

Freud, S. 1975 (1905). Tre saggi sulla teoria sessuale. In Opere di Sigmund Freud, IV, Torino: Bollati Boringhieri. 
Freud, S. 1976 (1915). Metapsicologia. In Opere di Sigmund Freud, VIII, Torino: Bollati Boringhieri.

Freud, S. 1977 (1919). Il perturbante. In Opere di Sigmund Freud, IX, Torino: Bollati Boringhieri.

Freud, S. 1978 (1925).La negazione. In Opere di Sigmund Freud, X, Torino: Bollati Boringhieri.

Geesey, P. 1997. "Cultural Hybridity and Contamination in Tayeb Salih's Season of Migration to the North." Research in African Literatures, 28: 128-40.

Genette, G. 1976 (1972). Figure III. Discorso del racconto. Torino: Einaudi.

Genette, G. 1997 (1982). Palinsesti. La letteratura al secondo grado. Torino: Einaudi.

Green, A. 2018 (1983). Narcisismo di vita, narcisismo di morte. Milano: Raffaello Cortina.

Hassan, W.S. 2003. "Gender (and) Imperialism: Structures of Masculinity in Tayeb Salih's Season of Migration to the North.” Men and Masculinities, 3: 309-24.

Holland, N. 1986 (1968). Le dinamiche della risposta letteraria. Bologna: Il Mulino. Husayn, Ț 2017 (1933). Adīb. Storia di un letterato. Venezia: Edizioni Ca' Foscari.

Iacono, A.M. 2000. Autonomia, potere, minorità. Del sospetto, della paura, della meraviglia, del guardare con altri occhi. Milano: Feltrinelli.

Jacobi, R. 1982. “The Camel-Section of the Panegyrical Ode.” Journal of Arabic Literature, 13: 1-22.

John, J., e Y. Tarawneh. 1988. "Tayeb Salih and Freud: the Impact of Freudian Ideas On Season of Migration To the North." Arabica, 35: 328-49.

Kafka, F. 1985 (1936). La partenza. In F. Kafka. I racconti. Milano: Rizzoli.

Laing, R.D. 1969 (1959). L'Io diviso. Studio di psichiatria esistenziale. Torino: Einaudi.

Mayer, S.G. 2001. The Experimental Arabic Novel. Postcolonial Literary Modernism in the Levant. New York: State University of New York Press.

Ogden, T.H. 1994. “The Analytic Third: Working with Intersubjective Clinical Facts.” The International Journal of Psychoanalysis 75: 3-19.

Ogden, T.H. 1999 (1994). Soggetti dell'analisi. Milano: Masson.

Ogden, T.H. 2013. L'orecchio dell'analista e l'occhio del critico. Ripensare psicoanalisi e letteratura. Milano: CIS editore.

Pioletti, A. 2014. La porta dei cronotopi. Tempo-spazio nella narrativa romanza. Soveria Mannelli: Rubettino.

Praz, M. 1966. La carne, la morte e il diavolo nella letteratura romantica. Firenze: Sansoni.

Rank, O. 2001 (1925). Il doppio. Uno studio psicoanalitico. Milano: SE, Milano.

Rovelli, C. 2020. Helgoland. Milano: Adelphi.

Saeed Adam, A.A. 2015. "Arevenge Endeavor (and) Unconscious Desire: Psychoanalytic study on Mustafa Saeed in Tayeb Salih's Season of Migration to the North." European Journal of English Language and Literature Studies 3: 95-102.

Said, E.W. 1999 (1978). Orientalismo. L'immagine europea dell'Oriente. Milano: Feltrinelli.

Said, E.W. 2002. Power, politics, and culture: Interviews with Edward W. Said. New York: Vintage.

Salih, T. 2011 (1967). La stagione della migrazione a Nord. Palermo: Sellerio, Palermo.

Sass, L.A. 2013 (1992). Follia e modernità. La pazzia alla luce dell'arte, della letteratura e del pensiero moderni. Milano: Raffaello Cortina.

Shaheen, M. 1985. “Tayeb Salih and Conrad.” Comparative Literature Studies 22: 156-71.

Siddiq, M. 1978. "The process of individuation in al-Tayyeb Salih's novel Season of Migration to the North." Journal of Arabic Literature 9: 67-104.

Thorne, K. 2019 (1994). Buchi neri e salti temporali. L'eredità di Einstein. Roma: Oblò Castelvecchi. 